\title{
Performance Monitoring in Supermarket Refrigeration Systems - Synchronization of Refrigerated Display Cases
}

\author{
Liang Chen ${ }^{*}$ Torben Green ${ }^{* *}$ Lars F. S. Larsen** \\ Rafael Wisniewski* Roozbeh Izadi-Zamanabadi** \\ * Department of Electronic Systems, Automation and Control, Aalborg \\ University, Fredrik Bajers Vej 7C, DK-9220 Aalborg, Denmark (email: \\ lchen.dhu@gmail.com,raf@es.aau.dk). \\ ** Danfoss A/S, Refrigeration and Air conditioning, DK-6430 \\ Nordborg, Denmark (email: Torben.Green@danfoss.com, \\ Lars.Larsen@danfoss.com,Roozbeh@danfoss.com).
}

\begin{abstract}
The overall task of a supermarket refrigeration system is to maintain the quality of the foodstuff. This is done by making use of a refrigeration cycle in which a refrigerant transport heat from the refrigerated display-cases to the outdoor surroundings. Typically the system is equipped with a decentralized control system neglecting interactions between subsystems. Though these interactions are minor they from time to time lead to a synchronization of the operation of the display-cases which causes an inferior control performance and increased energy consumption. In this paper we will analyze the synchronization using bifurcation theory and show that the system has a chaos-like behavior when it is not synchronized. Therefore, it is a good choice to de-synchronize the system by making the system chaotic. The positive maximal Lyapunov exponent is usually taken as an indication that the system is chaotic, it is used in this paper as a measure of performance for the tendency of the system to synchronize.
\end{abstract}

Keywords: Refrigeration system, Chaotic behavior, Hybrid system, Performance monitoring, Complex system.

\section{INTRODUCTION}

A supermarket refrigeration system consists of a central compressor rack that maintains the required flow of refrigerant to the refrigerated display cases located in the supermarket sales area. Each display case has an inlet valve for refrigerant that needs to be opened and closed such that the air temperature in the display case is kept within tight bounds to ensure a high quality of the goods. For many years, the control of supermarket refrigeration systems has been based on distributed control systems, which are flexible and simple. For example, each display case used to be equipped with an independent hysteresis controller that regulates the air temperature in the display case by manipulating the inlet valve. The major drawback, however, is that the control loops are vulnerable to selfinflicted disturbances caused by the interaction between the distributed control loops. In particular, practice and simulations show that the distributed hysteresis controllers have the tendency to synchronize [Larsen (2006)], meaning that the opening and closing actions of the valves coincide. Consequently, the compressor periodically has to work hard to keep up the required flow of refrigerant, which results in low efficiency, inferior control performance and a high wear on the compressor. The control problem is significantly complicated by the fact that many of the control inputs are restricted to discrete values, such as the opening/closing of the inlet valves and the stepwise control of the compressor. Furthermore, the system features switched dynamics turning the supermarket refrigeration system into a hybrid system.

The intense focus on limiting energy consumption and the global environmental awareness calls for energy efficient solutions. By monitoring the performance of the refrigeration system the "goodness" of the operation can be measured and early warnings about undesired behaviors can be given such that the control system can accommodate these and continuously optimize the system performance. In this paper we will focus on the monitoring of the synchronization phenomenon. By analyzing the system behavior using bifurcation and chaos theory [Crawford (1991), Devaney (2003)] it can be shown that the system has a chaos-like behavior. Bifurcation and chaos theory is most commonly applied to the mathematical study of dynamical systems to investigate dramatic changes in the qualitative or topological structure of a system. It can be dated back to 1975 when the first mathematical definition of 'chaos' was given [Li and Yorke (1975)]. The synchronization in the paper can be interpreted as a low order limit cycle [Wisniewski and Larsen (2008)]. It will be shown how the system jumps between low order and high order limit cycles varying the hysteresis bounds of the temperature controller. If the system converges towards a 
low order limit cycle it can be seen as an indication of a risk that the system may synchronize. Therefore, it is a good choice to de-synchronize the system by making the system chaotic. The positive maximal Lyapunov exponent is usually taken as an indication that the system is chaotic. A huge number of references are available on calculation of the Lyapunov exponents [Müller (1995); Benettin et al. (1980); Wolf et al. (1985)]. We suggest using the maximal Lyapunov exponent as a measure of performance for the tendency of the system to synchronize.

\section{SYSTEM DESCRIPTION}

The overall task for a supermarket refrigeration system is to maintain the quality of the stored foodstuff. The goods are usually stored in open display cases in the sales area of the supermarket. The working principle of the supermarket system is a refrigeration cycle which utilizes a refrigerant to transport heat from the display cases to the outdoor surroundings.

A simplified supermarket refrigeration circuit is shown in Fig. 1. The compressors and the display cases are in the majority of supermarket refrigeration systems connected in parallel. The compressors supply the flow by compressing the low pressure refrigeration which is drained from the suction manifold. The refrigerant then passes through the condenser and into the liquid manifold. Each display case has an expansion valve which is connected to the liquid manifold where from the refrigerant then flows through the expansion valve and into the evaporator of the display case. In the evaporator the refrigerant absorbs heat from the stored goods and thereby changes phase. The vaporized refrigerant flows into the suction manifold, thus closing the refrigerant cycle. The typical layout of a

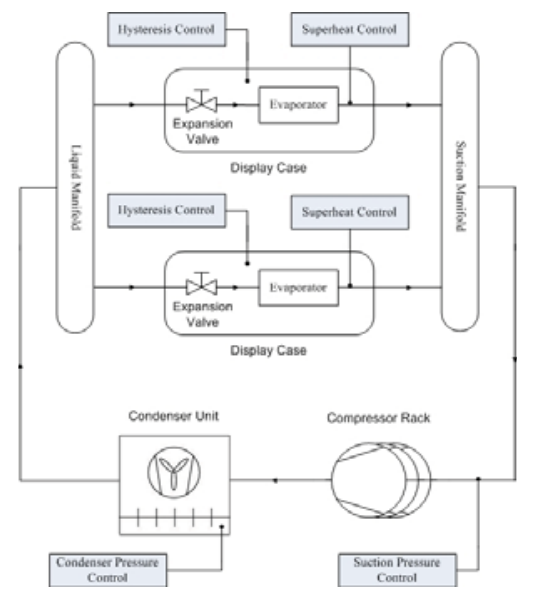

Fig. 1. Simplified supermarket refrigeration layout

display case can be seen in Fig. 2. The refrigerant is fed into the evaporator at the bottom of the display case and as the air is passed over the surface of the evaporator heat is absorbed which render a vaporization of the refrigerant. The resulting air flow creates an air curtain of cold air over the stored good. The air takes up the heat flow $\dot{Q}_{\text {goods-air }}$ from the stored goods and as a side effect the heat flow $\dot{Q}_{\text {load }}$ from the surroundings. The temperature of the air, $T_{\text {air }}$, is measured by a sensor mounted in the inlet air stream to the evaporator the goods to provide an indirect measure for the temperature of the goods.

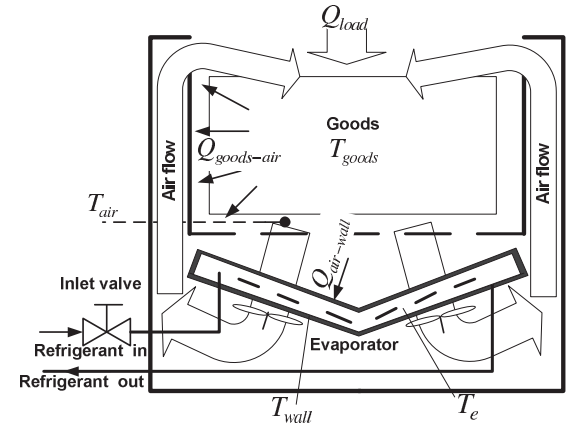

Fig. 2. Cross sectional view of a refrigerated display case.

\subsection{Traditional Control}

The typical controller structure for a supermarket refrigeration system is decentralized. Each of the display cases is fitted with an air temperature controller and a superheat controller, which ensures the desired filling of the evaporator. The compressor rack controls the suction pressure controller and the condenser fans control the condensing pressure. The only controllers considered in this paper is the suction pressure controller and the temperature controllers in the display cases.

The temperature in the display cases is controlled by a hysteresis controller that opens and closes the expansion valve, i.e. the valve opens when $T_{\text {air }}$ reaches a predefined upper temperature limit and stay open until $T_{\text {air }}$ decreases to the lower temperature limit and the valve closes again.

The suction pressure is controlled by switching compressors in the compressor rack on or off. A dead band around the reference is introduced to avoid excessive switching of the compressors. If the pressure exceeds the upper bound of the dead band one or more compressors are switched on. If the pressures drops below the lower bound of the dead band a compressor is switched off. This control strategy prevents moderate changes in the suction pressure from initiating compressor switching.

In a common supermarket many of the display cases will be alike and in addition be working under the same conditions. Thus, the switching frequency, for each of the expansion valves for the different display cases, will be close to each other. The display cases have a tendency to synchronize because there individual dynamics are coupled through the suction pressure. Synchronization of the display cases lead to periodic high and low amount of vaporized refrigerant flow into the suction manifold. Hence, large fluctuations in the suction pressure will be a consequence which then leads to higher switch frequency of the compressors and therefore excessive wear on the compressors. The result from synchronizing display cases can be seen in Fig. 3

\section{MODEL OF THE REFRIGERATION SYSTEM}

The model for the supermarket refrigeration system is composed of a number of sub-models which each represent a component in the refrigeration system. That is, individual models are made for the display cases, the suction manifold, the compressor rack, and the condensing unit. Because the emphasis of the paper is to examine 


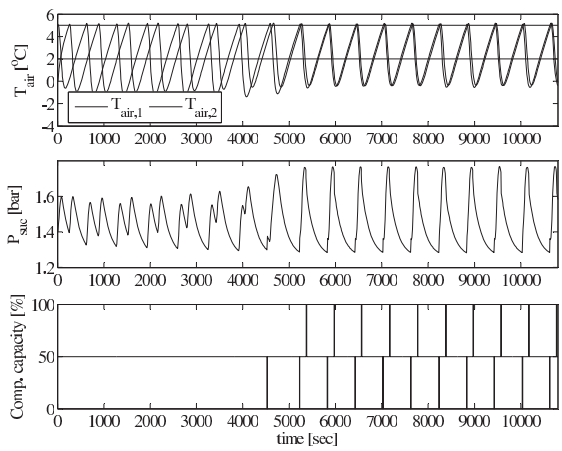

Fig. 3. The effect of synchronization

the synchronization phenomenon the modeling will be concentrated on the display cases and the suction manifold such that only the dynamics relevant for the control of the hysteresis control and the compressors are captured. The dynamic of the compressors are usually much faster than the dynamics of the rest of the refrigeration system. Thus, the modeling of the compressors dynamic is neglected.

The mathematical model presented in this section is a summary of the supermarket refrigeration model developed in [Larsen et al. (2007)]. The suction pressure $P_{\text {suc }}$, comprises the common state for the combined models. Each display case, $i=1, \ldots, N$ where $N$ is the number of display cases in the system, is described by four states. That is, the temperature of the goods $T_{\text {goods.i }}$, the air temperature $T_{\mathrm{air}, \mathrm{i}}$, the temperature of the evaporator wall $T_{\text {wall, } \mathrm{i}}$, and the mass of the refrigerant in the evaporator $M_{r, i}$. The input is the volumetric flow generated by the compressors $\dot{V}_{\text {comp }}$, and the binary state of the $i$ th inlet valve $\delta_{i}$ (closed or opened, $\delta_{i} \in\{0,1\}$ ). The systems are affected by the heat load from the surroundings of the display case $\dot{Q}_{\text {load }}$.

$$
\begin{aligned}
& \frac{\mathrm{d} T_{\text {goods,i }}}{\mathrm{d} t}=-\frac{\dot{Q}_{\text {goods-air,i }}(\cdot)}{M_{\text {goods }, \mathrm{i}} C_{\mathrm{p}, \text { goods }, \mathrm{i}}} \\
& \frac{\mathrm{d} T_{\text {wall, } \mathrm{i}}}{\mathrm{d} t}=\frac{\dot{Q}_{\text {air }- \text { wall }, \mathrm{i}}(\cdot)-\dot{Q}_{\mathrm{e}, \mathrm{i}}(\cdot)}{M_{\text {wall }, \mathrm{i}} C_{\mathrm{p}, \text { wall,i }}} \\
& \frac{\mathrm{d} T_{\text {air }, \mathrm{i}}}{\mathrm{d} t}=\frac{\dot{Q}_{\text {goods-air }, \mathrm{i}}(\cdot)+\dot{Q}_{\text {load }, \mathrm{i}}(\cdot)-\dot{Q}_{\text {air-wall }, \mathrm{i}}(\cdot)}{M_{\text {air }} C_{\mathrm{p}, \text { air }, \mathrm{i}}} \\
& \frac{\mathrm{d} M_{r, i}}{\mathrm{~d} t}= \begin{cases}\frac{M_{\mathrm{r}, \max , \mathrm{i}}-M_{\mathrm{r}, \mathrm{i}}}{\tau_{\text {fill, }}} & \text { if } \delta_{i}=1 \\
-\frac{\dot{Q}_{\mathrm{e}, \mathrm{i}}(\cdot)}{\Delta h_{\mathrm{lg}}\left(P_{\text {suc }}\right)} & \text { if } \delta_{i}=0 \text { and } M_{\mathrm{r}, \mathrm{i}} \geq 0 \\
0 & \text { if } \delta_{i}=0 \text { and } M_{\mathrm{r}, \mathrm{i}}=0\end{cases} \\
& \frac{\mathrm{d} P_{\text {suc }}}{\mathrm{d} t}=\frac{\dot{m}_{\mathrm{in}-\mathrm{suc}}(\cdot)+\dot{m}_{\mathrm{r}, \mathrm{const}}-\dot{V}_{\mathrm{comp}} \rho_{\mathrm{suc}}\left(P_{\mathrm{suc}}\right)}{V_{\text {suc }} \nabla \rho_{\mathrm{suc}}\left(P_{\mathrm{suc}}\right)}
\end{aligned}
$$

The enthalpy difference across the two-phase region of the evaporator is denoted by $\Delta h_{\mathrm{lg}}$, the density of the refrigerant is denoted by $\rho_{\text {suc }}$, and $\nabla \rho_{\text {suc }}$ denotes the pressure derivative of the refrigerant density. $T_{e}$ is the evaporation temperature for the refrigerant. The subscripts for the mass $M$ and the heat capacity $C_{p}$ denotes the media. The heat flow is denoted by $\dot{Q}$ where the subscript indicates the media between which the thermal energy is exchanged. In addition, the varies heat flows are defined by the following functions:

$$
\begin{gathered}
\dot{Q}_{\text {goods-air }, \mathrm{i}}\left(T_{\text {goods }, \mathrm{i}}, T_{\text {air }, \mathrm{i}}\right)= \\
U A_{\text {goods-air }, \mathrm{i}} \cdot\left(T_{\text {goods }, \mathrm{i}}-T_{\text {air }, \mathrm{i}}\right) \\
\dot{Q}_{\text {air-wall,i }}\left(T_{\text {air }, \mathrm{i}}, T_{\text {wall }, \mathrm{i}}\right)=U A_{\text {air-wall }, \mathrm{i}} \cdot\left(T_{\text {air }, \mathrm{i}}-T_{\text {wall }, \mathrm{i}}\right) \\
\dot{Q}_{e, i}\left(M_{r, i}, T_{\text {wall }, \mathrm{i}}, P_{\text {suc }}\right)= \\
U A_{\text {wall-ref }, \mathrm{i}}\left(M_{r, i}\right) \cdot\left(T_{\text {wall }, \mathrm{i}}-T_{e}\left(P_{\text {suc }}\right)\right) \\
U A_{\text {wall-ref }, \mathrm{i}}\left(M_{r, i}\right)=U A_{\text {wall-ref }, \text { max }, \mathrm{i}} \cdot \frac{M_{\mathrm{r}, \mathrm{i}}}{M_{\mathrm{r}, \text { max }, \mathrm{i}}}
\end{gathered}
$$

The overall heat transfer coefficient is denoted by $U A$ and the subscript denotes the media from which the heat is transferred. In addition the mass flow rate in the suction manifold is given by:

$$
\dot{m}_{\mathrm{in}-\mathrm{suc}}\left(M_{\mathrm{r}, \mathrm{i}}, T_{\mathrm{wall}, \mathrm{i}}, P_{\mathrm{suc}}\right)=\sum_{i=1}^{N} \frac{\dot{Q}_{\mathrm{e}, \mathrm{i}}(\cdot)}{\Delta h_{\mathrm{lg}}\left(P_{\mathrm{suc}}\right)}
$$

The functions $\Delta h_{\mathrm{lg}}, \rho_{\mathrm{suc}}$, and $T_{e}$ are refrigerant specific. Detailed description of these functions are given in [Larsen et al. (2007)]. In (4) it can be seen that the system have a hybrid nature due to the the discrete input which represents the opening and closing of the expansion valves.

\subsection{Simplified model}

In order to obtain a model that is suitable for analyzing the synchronization phenomenon the equation system (1) through (5) are further simplified to a second order affine switched system.

The simplification of the model is based on the following assumptions:

- The heat capacity of the goods is large, thus the temperature of the goods in a display case is constant and equal $T_{\mathrm{g} 0}$.

- The heat capacity of the air is small.

- The evaporator is instantly filled (emptied) when the inlet valve is opened (closed).

- The mass flow out of the display case when the valve is open is constant and equal $\dot{m}_{0}$.

- The evaporation temperature $T_{\mathrm{e}}$ and the density $\rho_{\text {suc }}$ of the refrigerant in the suction manifold are affine functions of suction pressure $P_{\text {suc }}$,

$$
T_{\mathrm{e}}=a_{T} P_{\mathrm{suc}}+b_{T} \text { and } \rho_{\mathrm{suc}}=a_{\rho} P_{\mathrm{suc}}+b_{\rho}
$$

- The gradient $\nabla \rho_{\text {suc }}\left(P_{\text {suc }}\right) \equiv \nabla \rho_{\text {suc } 0}\left(P_{\text {suc } 0}\right)$ is constant.

- The compressor delivers a constant volume flow $\dot{V}_{\text {comp }}$.

- The heat load $\dot{Q}_{\text {load }}$ on the display cases is constant.

Based on these assumptions the dynamic of the air temperature $T_{\text {air,i }}$ in the $i$ th display case can be formulated as follows: 


$$
\begin{aligned}
\frac{\mathrm{d} T_{\text {air }, \mathrm{i}}}{\mathrm{d} t} & =\frac{\dot{Q}_{\text {goods-air,i }}+\dot{\mathrm{Q}}_{\text {load }, \mathrm{i}}-\delta_{i} \dot{\mathrm{Q}}_{\mathrm{e}, \text { max }, \mathrm{i}}}{\left(1+\frac{U A_{\text {goods }- \text { air }, \mathrm{i}}}{U A_{\text {air }- \text { wall }, \mathrm{i}}}\right) M_{\text {wall }, \mathrm{i}} C p_{\text {wall }, \mathrm{i}}} \\
T_{\text {wall }, \mathrm{i}} & =T_{\text {air }, \mathrm{i}}-\frac{\dot{Q}_{\text {goods-air }, \mathrm{i}}+\dot{Q}_{\text {load }, \mathrm{i}}}{U A_{\text {air }- \text { wall }, \mathrm{i}}} \\
\dot{Q}_{\text {goods }- \text { air }, \mathrm{i}} & =U A_{\text {goods-air }, \mathrm{i}}\left(T_{\mathrm{g} 0, \mathrm{i}}-T_{\text {air }, \mathrm{i}}\right) \\
\dot{Q}_{\mathrm{e}, \text { max }, \mathrm{i}} & =U A_{\text {wall }} \text {-ref }, \text { max }, \mathrm{i}\left(T_{\text {wall }, \mathrm{i}}-a_{T} P_{\text {suc }}-b_{T}\right),
\end{aligned}
$$

The suction manifold dynamics is governed by the expression

$$
\frac{\mathrm{d} P_{\mathrm{suc}}}{\mathrm{d} t}=\frac{\sum_{i=1}^{N} \delta_{i} \dot{m}_{0, \mathrm{i}}+\dot{m}_{\mathrm{r}, \mathrm{const}}-\dot{V}_{\mathrm{comp}}\left(a_{\rho} P_{\mathrm{suc}}+b_{\rho}\right)}{V_{\mathrm{suc}} \cdot \nabla \rho_{\mathrm{suc} 0}} .
$$

Thus, the non-linear hybrid system has been reduced to a two order (for each display case) affine system with discrete inputs. For a refrigeration system with two display cases, the system states of the simplified model are $T_{\text {air,i }}(i=1,2)$ and $P_{\text {suc }}$. The discrete inputs are $\delta_{i} \in\{0,1\}$, which indicate if the valves are closed or open. The input $\delta$ is controlled by a hysteresis controller which changes the value of $\delta$ in the following way:

$$
\delta_{i}(k+1)= \begin{cases}1 & \text { if } T_{a i r, i} \geq \overline{T_{\text {air }, \mathrm{i}}} \\ 0 & \text { if } T_{a i r, i} \leq T_{\mathrm{air}, \mathrm{i}} \\ \delta_{i}(k) & \text { if } \underline{T_{\mathrm{air}, \mathrm{i}}}<\overline{T_{a i r, i}}<\overline{T_{\mathrm{air}, \mathrm{i}}},\end{cases}
$$

where $k$ denotes the time index, $\overline{T_{a i r}, i}$ is the upper bound the air temperature and $T_{a i r, i}$ is the lower bound .

\section{DYNAMICAL ANALYSIS}

In this section, we will analyze dynamics of the simplified refrigeration model through bifurcation and chaos theory. The theory is most commonly applied to the mathematical study of dynamical systems. The aim of the theory is to investigate dramatic changes in the qualitative or topological structure of a system by changing smoothly a system parameter. For the refrigeration system we will analyze the system behavior by changing smoothly the lower bound in one of the display cases. The resulting behavior will be depicted in a so-called bifurcation diagram, from which the synchronization phenomenon will be studied, thereafter a measure will be developed to evaluate the tendency of synchronization. All simulation results in the section are based on the following parameter settings:

\subsection{Phase plots w.r.t $\underline{T_{\text {air }, 2}}$}

We shall study bifurcation, i.e. the influence of changes of system parameters on the system behavior at large. There are two system parameters, i.e. the upper bound and the lower bound of the temperature in the air temperature control of the display case. Here, we examine the lower bound of the second display case $T_{\mathrm{air}, 2}$. This will provide an example which will help understand how the system

\begin{tabular}{|c|c|c|c|c|c|}
\hline \multicolumn{6}{|l|}{ Display cases } \\
\hline$U A_{\text {wall-ref,max }}$ & & $\frac{J}{s \cdot K}$ & $T_{\mathrm{g} 0}$ & 3.0 & ${ }^{0} \mathrm{C}$ \\
\hline$U A_{\text {goods-air }}$ & 300 & $\frac{s \cdot f^{A}}{s \cdot K}$ & $\dot{m}_{0}$ & 1.0 & $\mathrm{~kg} / \mathrm{s}$ \\
\hline$U A_{\text {air-wall }}$ & 500 & $\frac{s \cdot f^{A}}{s \cdot K}$ & $\dot{Q}_{\text {load }}$ & 3000 & $\mathrm{~J} / \mathrm{s}$ \\
\hline$\dot{m}_{\mathrm{r}, \text { const }}$ & & $\frac{\mathrm{kg}}{\mathrm{s}}$ & $M_{\text {wall }}$ & 260 & $\mathrm{~kg}$ \\
\hline$\nabla \rho_{\text {suc0 }}$ & 4.6 & $\frac{{ }^{s} \mathrm{~kg}}{\mathrm{~m}^{3} \mathrm{bar}}$ & $C_{\mathrm{p}, \text { wall }}$ & 385 & $\frac{\mathrm{J}}{\mathrm{kg} \cdot \mathrm{K}}$ \\
\hline \multicolumn{6}{|c|}{$\begin{array}{l}\text { The same parameters has been used for all disp. } \\
\text { Compressor }\end{array}$} \\
\hline$\dot{V}_{\text {comp }}$ & 0.28 & $\frac{m^{3}}{s}$ & & & \\
\hline \multicolumn{6}{|c|}{ Suction manifold } \\
\hline$V_{\text {suc }}$ & 5.00 & $m^{3}$ & & & \\
\hline \multicolumn{6}{|c|}{ Air temperature control } \\
\hline$T_{\text {air }, \mathrm{i}}$ & 0.00 & ${ }^{0} \mathrm{C}$ & $\overline{\overline{T_{\text {air }, \mathrm{i}}}}$ & 5.00 & ${ }^{0} \mathrm{C}$ \\
\hline \multicolumn{6}{|l|}{$\overline{i \text { for the disp. }}$} \\
\hline \multicolumn{6}{|l|}{ Coefficients } \\
\hline$a_{T}=16.2072$ & & $b_{T}=41.9095$ & $a_{\rho}=4.6$ & & $b_{\rho}=0.4$ \\
\hline
\end{tabular}
behaves with the varying parameter.

Fig. 4 shows some typical phase plots of the system states $T_{\mathrm{air}, 1}$ and $T_{\mathrm{air}, 2}$. When the parameter $\underline{T_{\mathrm{air}, 2}}=0$,
Table 1. Parameters for a simplified supermarket refrigeration system

the limiting behavior of the system switches between the two points $(0,0)$ and $(5,5)$ within an accepted tolerance $(1 E-6$ in the paper). Here, we call it as a 2-periodic limit cycle. The period of a limit cycle is defined by the sum of a number of switching points on the boundary $\partial \square=\partial\left(\left[T_{\mathrm{air}, 1}, \overline{T_{\mathrm{air}, 1}}\right] \times\left[T_{\mathrm{air}, 2}, \overline{T_{\mathrm{air}, 2}}\right]\right)$. The phase plot of the 2-periodic limit cycle corresponds to the synchronization phenomenon mentioned in the above section, where the two states $T_{\mathrm{air}, 1}$ and $T_{\mathrm{air}, 2}$ agree all the time. When the parameter $T_{\mathrm{air}, 2}$ increases slightly to the value of 0.15 , a 4-periodic limit cycle appears, which is similar to the synchronization but with a bigger difference of the two states; we will call it the quasi-synchronization. When $\underline{T_{\text {air }, 2}}=0.2$, another topology of 4-periodic limit cycle appears in the phase plot, which is totally different from the state agreement in the synchronization. If we continue increasing $T_{\mathrm{air}, 2}$ to the value of 0.3 , we will find that the system tends to a high-periodic limit cycle with many switching points in the boundary of $\partial \square$. It looks like chaos, the common phenomenon in the nonlinear system [Devaney (2003)]. It is far away from the synchronization.
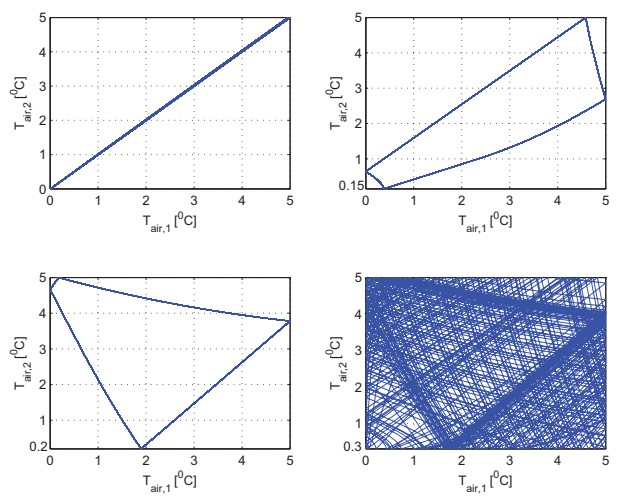

Fig. 4. Typical limiting behaviors with the various values of the parameter $T_{\text {air }, 2}$. (a) 2-periodic limit cycle (synchronization, $\overline{T_{\mathrm{air}, 2}}=0$ ), (b) 4-periodic limit cycle $\left(T_{\text {air }, 2}=0.15\right),(\mathrm{c})$ another 4-periodic limit cycle $\left(T_{\mathrm{air}, 2}=0.2\right),(\mathrm{d})$ high-periodic limit cycle $\left(T_{\mathrm{air}, 2}=\right.$ $0.3)$. 
Synchronization of the display cases leads to large fluctuations in the suction pressure which then result in higher switch frequency of the compressors. It reduces lifetime of the compressors and enlarges energy consumption. Fig. 5 shows the comparison of the suction pressures between the synchronization and the chaos-like situation. We can see that in the chaos-like situation, the fluctuation range of the suction pressure decreases occasionally; even for the part with the same fluctuation amplitude as the synchronization, the pressure jumps so fast that the traditional PI controller in the compressor can fix it. Therefore, we conclude that good control performance can be achieved if the system behaves like chaos.

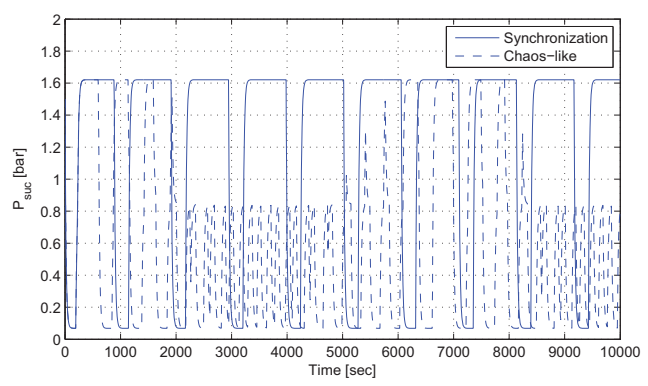

Fig. 5. The suction pressure $P_{\text {suc }}$ in the situations of (a) synchronization and (b) chaos-like $\left(T_{\mathrm{air}, 2}=0.3\right)$.

\subsection{Bifurcation diagram w.r.t $T_{\mathrm{air}, 2}$}

To show how the system behaves with the smooth change of the parameter, we usually use the bifurcation diagram. A bifurcation diagram exhibits the possible long-term values (equilibria/fixed points or periodic orbits) of a system as a function of parameters in the system. A bifurcation occurs when a small smooth change made to the values of the bifurcation parameter causes a sudden 'qualitative' or topological change in its behavior. In this paper, the lower bound of the second display case $T_{\text {air,2 }}$ is considered as the bifurcation parameter. Fig. 6 shows the limiting behavior of the state $T_{\text {air,1 }}$ with respect to the parameter $T_{\mathrm{air}, 2}$. The system exhibits very complicated behaviors. When $\bar{T}_{\text {air }, 2}=0$, the system stabilizes at a 2 periodic limit cycle with the phase plot shown in Fig. 4a. As the parameter $T_{\text {air,2 }}$ increases, the 2-periodic limit cycle becomes unstable, and a stable 4-periodic limit cycle appears (the phase plot is like Fig. 4b). The limit cycle retains the similar shape until another stable 4-periodic limit cycle occurs at $T_{\text {air,2 }}=0.186$ (the phase plot is like Fig. 4c). The shape $\overline{\text { keeps }}$ until $T_{\text {air }, 2}=0.234$, a higher order oscillation, like chaos, is generated (the phase plot is like Fig. 4d). If the parameter continuously rise, we can see that the system behavior becomes very complex and keeps switching between order and chaos-like oscillations.

The bifurcation diagram demonstrates that the simple refrigeration model is very sensitive to a change in the parameter $T_{\mathrm{air}, 2}$. If we change the parameter slightly, we obtain a totally different topology of the behavior. Therefore, we may ask: is it possible to suppress the synchronization phenomenon we found in the practice of the supermarket refrigeration system by adjusting a little

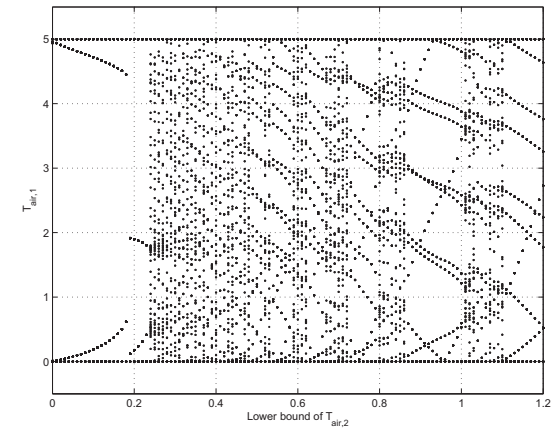

Fig. 6. Bifurcation diagrams of the system state $T_{\text {air, } 1}$ w.r.t the parameter $\underline{T_{\text {air }, 2}}$.

bit value of the bounds $\overline{T_{\text {air }}}$ or $T_{\text {air }}$ ? It is obvious for a small 'toy' system with only two display cases that by changing the bound of the temperature, the system can be de-synchronized by making it chaotic. However, for a real plant with many different display cases, it is not obvious how to select the bounds. Hence, we are seeking a method to identify whether the bounds are selected such that the system will synchronize or not. For this purpose we will use the maximal Lyapunov exponent.

\subsection{Description of Lyaponov exponent}

The Lyapunov exponent characterizes the averaged rate of separation of two close trajectories in the phase space [Oseledec (1968)]. Quantitatively, two trajectories $x_{1}(t)$ and $x_{2}(t)$ in phase space with initial separation $\delta x(0)$ diverge

$$
|\delta x(t)| \approx e^{\lambda t}|\delta x(0)|
$$

where $\delta x(t)=x_{1}(t)-x_{2}(t), \lambda$ is the Lyapunov exponent. The negative Lyapunov exponent measures the exponential convergence of trajectories, and the positive measures the exponential divergence of trajectories.

There are $n$ Lyapunov exponents in the spectrum of an $n$ dimensional dynamical system. It is common to just refer to the largest one, i.e. the maximal Lyapunov exponent (MLE), which is defined as follows:

$$
\lambda_{\max }=\lim _{t \rightarrow \infty} \frac{1}{t} \ln \frac{|\delta x(t)|}{|\delta x(0)|} .
$$

The positive MLE is usually taken as an indication that the system is chaotic. It is used in this paper as a measure of performance for the tendency of the system to synchronize, that is, the higher value of the MLE the lower risk for synchronization.

\subsection{Algorithm for computation the MLE}

Algorithms for computing the Lyapunov exponents of the "smooth" dynamical system are well established [Benettin et al. (1980), Wolf et al. (1985)]. Let us consider the system

$$
\dot{x}=f(x(t)) \text {, }
$$

where $f \in C^{1}$ is a continuously differentiable vector function with the initial condition $x\left(t_{0}\right)=x(0)$. The algorithm is based on the integration of the linearized equation (20) as follows:

$$
\delta \dot{x}=J(t) \delta x,
$$


where

$$
J(t)=\left.\frac{\partial f(x)}{\partial x^{T}}\right|_{x=x(t)}
$$

is the Jacobian matrix of $f$ w.r.t the trajectory under consideration. The MLE $\lambda_{\max }$ is given as the average for some different initial conditions $\delta x(0)$ as Eq. (18).

For the "non-smooth" dynamical system with discontinuities like the refrigeration system in this paper, the above algorithm cannot be directly applied. One way to calculate the MLE is to supplement the transition conditions at the instants of discontinuities into the linearized equations [Müller (1995)]. This model-based method requires exact system information and the calculation complexity greatly increases with the number of the sub-systems. It is not suitable for the refrigeration system especially with many display cases in the supermarket. Besides, to apply this method one needs to know the switching sequence of the sub-systems in advance. It is also impossible in our case. We prefer to take the practical view of calculating the MLE from experimental data. Two well-known papers are [Wolf et al. (1985), S. Sato and Sawada (1987)]. Basic computing steps are given in the following:

(1) Based on the $N$-point time series $x_{1}, x_{2}, \ldots, x_{N}$, reconstruct the phase space:

$$
Y_{i}=\left[x_{i}, x_{i+\tau}, \ldots, x_{i+(m-1) \tau}\right] \in R^{n}(i=1, \ldots, M),
$$

where $M=N-(m-1) \tau, \tau$ is the reconstruction delay, $m$ is the embedding dimension.

(2) Find the nearest neighbor, $Y_{\hat{i}}$, by searching for the point that minimizes the distance to the particular reference point $Y_{i}$, that is, $d_{i}(0)=\min _{Y_{\hat{i}}}\left\|Y_{i}-Y_{\hat{i}}\right\|$. After $j$ discrete-time steps, the distance $d_{i}(0)$ goes to $d_{i}(j)$.

(3) Estimate the averaged rate of distance separation as the MLE:

$$
\lambda_{\max }=\frac{1}{j \cdot \Delta t} \cdot \frac{1}{(M-j)} \sum_{i=1}^{M-j} \frac{d_{i}(j)}{d_{i}(0)},
$$

where $\Delta t$ is the sampling period of the time series.

\section{CONCLUSION}

The main focus of this paper was on dynamic analysis of a simple system with two display cases by using bifurcation and chaos theory. Interpreting synchronization as a low periodic limit cycle and by varying the hysteresis bounds of the temperature controller it was shown that the system exhibited a complex chaos-like behavior when it was not synchronized, i.e. it switches between low and high periodic limit cycles. Synchronization of the system has proven to result in an inferior performance due to the resulting large pressure variations. It was, however, indicated in this paper that by de-synchronizing the system (by making it chaotic) it is possible to significantly reduce the pressure variations and hence improve the performance. The positive maximal Lyapunov exponent, usually used as the indication of chaos, was in this paper used as a measure of performance for the tendency of the system to synchronize. These findings for the small "toy" system may seem obvious, however they can easily be scaled to (realistic) large scale systems, where it is harder to distinguish and evaluate "good" and "bad" behavior.

\section{REFERENCES}

Benettin, G., Galgani, L., Giorgilli, A., and Strelcyn, J.M. (1980). Lyapunov characteristic exponents for smooth dynamical systems and for hamiltonian systems; a method for computing all of them. part i: Theory, part ii: Numerical application. Meccnnica, 15:9-20, 21-30.

Crawford, J.D. (1991). Introduction to bifurcation theory. Reviews of Modern Physics, Vol. 63, No. 4, 991-1037.

Devaney, R.L. (2003). An Introduction to Chaotic Dynamical Systems. Westview Press.

Georgiou, I.T. and Schwartz, I.B. (1999). Dynamics of large scale coupled structural/ mechanical systems: A singular perturbation/proper orthogonal decomposition approach. SIAM J. Appl. Math., 59:1178-1207.

Kennedy, M.P., Kolumban, G., and Kis, G. (2000). Chaotic modulation for robust digital communications over multipath channels. Int. J. Bifur. Chaos, 10:695718.

Larsen, L.F.S. (2006). Model Based Control of Refrigeration Systems. Ph.D. thesis, Department of Control Engineering - Aalborg University, Aalborg.

Larsen, L., Izadi-Zamanabadi, R., Wisniewski, R., and Sonntag, C. (2007). Supermarket refrigeration systems - a benchmark for the optimal control of hybrid systems. Technical report, Hycon, http://astwww.bci.unidortmund.de/hycon4b/wprelated/sr.pdf.

Li, T.Y. and Yorke, J.A. (1975). Period three implies chaos. Amer. Math. Monthly, 82:481-485.

Müller, P.C. (1995). Calculation of lyapunov exponents for dynamic systems with discontinuities. Chaos, Solitons and Fractals, 9:1671-1681.

Oseledec, V.I. (1968). A multiplicative ergodic theorem: Lyapunov characteristic numbers for dynamical systems. Trans. Moscow Math. Soc., 19:197-231.

Ottino, J.M., Muzzio, F.J., and Tjahjadi, M. (1992). Chaos, symmetry, and self-similarity: Exploiting order and disorder in mixing processes. Science, 257:754-760.

S. Sato, M.S. and Sawada, Y. (1987). Practical methods of measuring the generalized dimension and the largest lyapunov exponent in high dimensional chaotic systems. Prog. Theor. Phys., 77:1.

Schiff, S.J., Jerger, K., and Duong, D.H. (1994). Controlling chaos in the brain. Nature, 370:615-620.

Wisniewski, R. and Larsen, L.F.S. (2008). Method for analysis of synchronization applied to supermarket refrigeration system. In 17 th IFAC World Congress. Seoul, Korea.

Wolf, A., Swift, J.B., Swinney, H.L., and Vastano, J.A. (1985). Determining lvapunov exponents from a time series. Physica D, 16:285-317. 